\title{
A two-sided analogue of the Coxeter complex
}

\author{
T. Kyle Petersen ${ }^{1} \|^{\dagger}$ \\ ${ }^{1}$ DePaul University, Chicago, IL, USA
}

\begin{abstract}
For any Coxeter system $(W, S)$ of rank $n$, we introduce an abstract boolean complex (simplicial poset) of dimension $2 n-1$ which contains the Coxeter complex as a relative subcomplex. Faces are indexed by triples $(J, w, K)$, where $J$ and $K$ are subsets of the set $S$ of simple generators, and $w$ is a minimal length representative for the double parabolic coset $W_{J} w W_{K}$. There is exactly one maximal face for each element of the group $W$. The complex is shellable and thin, which implies the complex is a sphere for the finite Coxeter groups. In this case, a natural refinement of the $h$-polynomial is given by the "two-sided" $W$-Eulerian polynomial, i.e., the generating function for the joint distribution of left and right descents in $W$.

Résumé. Pour tout système de Coxeter $(W, S)$ de rang $n$, nous introduisons un complexe booléen (poset simplicial) de dimension $2 n-1$ qui contient le complexe de Coxeter comme sous-complexe relatif. Les faces sont indexées par les triplets $(J, w, K)$, où $J$ et $K$ sont des sous-ensembles de l'ensemble $S$ de générateurs simples, et $w$ est un représentant de la longueur minimale pour le double coset parabolique $W_{J} w W_{K}$. Il y a exactement une face maximale pour chaque élément du groupe $W$. Le complexe est épluchanble et maigre, ce qui implique que le complexe est une sphère pour les groupes de Coxeter finis. Dans ce cas, un raffinement naturel du $h$-polynomial est donné par le polynôme $W$-Eulérien "deux côtés", à savoir, la fonction génératrice pour la distribution conjointe des descentes gauche et à droite dans $W$.
\end{abstract}

Keywords. Coxeter group, Coxeter complex, Eulerian polynomial, contingency table

\section{Introduction}

Coxeter groups were developed to study symmetries of regular polytopes, and they play a major role in the study of Lie algebras (the Weyl group of a root system is a Coxeter group). The Coxeter complex is a simplicial complex associated with the reflection representation of the group, but which can also be defined abstractly via cosets of parabolic subgroups. The goal of this paper is to provide a "two-sided" analogue of the Coxeter complex by considering double cosets of parabolic subgroups.

Before turning to the new construction, let us recall some definitions and important properties of the usual Coxeter complex. We assume the reader has some familiarity with the study of Coxeter groups. See Humphreys' book [14] or Björner and Brenti's book [6] for background.

Fix a finitely generated Coxeter system $(W, S)$, and let $W_{J}$ denote the standard parabolic subgroup generated by a subset of simple generators $J \subseteq S$. It is well known that the set of cosets of parabolic subgroups forms an abstract simplicial complex known as the Coxeter complex, and denoted by

$$
\Sigma=\Sigma(W, S)=\left\{w W_{J}: w \in W, J \subseteq S\right\} .
$$

\footnotetext{
$\dagger^{\dagger}$ Email: tpeter21@ depaul . edu. Supported by a Simons foundation collaboration grant. 
The faces of $\Sigma$ are ordered by reverse inclusion of cosets, i.e.,

$$
w W_{J} \leq_{\Sigma} w^{\prime} W_{J^{\prime}} \quad \text { if and only if } \quad w W_{J} \supseteq w^{\prime} W_{J^{\prime}}
$$

Note that this means maximal elements are singleton sets: $w W_{\emptyset}=\{w\}$, and there is a unique minimal element: $w W_{S}=W$. Some well-known features of the Coxeter complex are highlighted in the following result, most of which can be found in work of Björner [5] (see also Abramenko and Brown [1, Chapter 3]), though most of these facts were known earlier. See, e.g., Bourbaki [7].

Theorem 1 For any Coxeter system $(W, S)$ with $|S|=n<\infty$ we have the following.

1. The Coxeter complex $\Sigma$ is a balanced simplicial complex of dimension $n-1$.

2. The facets (maximal faces) of $\Sigma$ are in bijection with the elements of $W$.

3. The Coxeter complex is shellable and any linear extension of the weak order on $W$ gives a shelling order for $\Sigma$.

4. If $W$ is infinite then $\Sigma$ is contractible.

5. If $W$ is finite,

(a) the geometric realization of $\Sigma$ is a sphere, and

(b) the h-polynomial of $\Sigma$ is the $W$-Eulerian polynomial,

$$
h(\Sigma ; t)=\sum_{w \in W} t^{\operatorname{des}(w)},
$$

where $\operatorname{des}(w)$ denotes the number of descents of the element $w$.

We will try to emulate all these properties for a "two-sided" version of the Coxeter complex, denoted $\Xi=\Xi(W, S)$. Our main results are summarized as follows.

Theorem 2 For any Coxeter system $(W, S)$ with $|S|=n<\infty$, we have the following.

1. The complex $\Xi$ is a balanced boolean complex of dimension $2 n-1$.

2. The facets (maximal faces) of $\Xi$ are in bijection with the elements of $W$, and the Coxeter complex $\Sigma$ is a relative subcomplex of $\Xi$.

3. The complex $\Xi$ is shellable and any linear extension of the two-sided weak order on $W$ gives a shelling order for $\Xi$.

4. If $W$ is infinite then $\Xi$ is contractible.

5. If $W$ is finite,

(a) the geometric realization of $\Xi$ is a sphere, 
(b) a refined h-polynomial of $\Xi$ is the two-sided $W$-Eulerian polynomial,

$$
h(\Xi ; s, t)=\sum_{w \in W} s^{\operatorname{des}_{L}(w)} t^{\operatorname{des}_{R}(w)},
$$

where $\operatorname{des}_{L}(w)$ denotes the number of left descents of $w$ and $\operatorname{des}_{R}(w)$ denotes the number of right descents of the element $w$.

The main contrasts between $\Xi$ and $\Sigma$ lie in the fact that $\Xi$ is roughly twice the dimension of $\Sigma$ and in the fact that $\Xi$ is not a simplicial complex. While all the faces of $\Xi$ are simplices, many of these simplices share the same vertex set.

We remark that our approach in this work is combinatorial, not geometric. There are two different approaches to proving the topological results for the Coxeter complex listed in Theorem 1 . One way (following Bourbaki [7]) is to relate the faces of the Coxeter complex to the faces of the reflection hyperplane arrangement for the Coxeter group. Thus the topology of the Coxeter complex is manifest in the ambient space. On the other hand, Björner showed in [5] how to use poset-theoretic tools to study the topology of the complex with only the abstract definition of the face poset.

The approach of this paper mirrors that of Björner. We define the face poset of $\Xi$ abstractly, and use Björner's techniques to deduce Theorem 2. We hope to uncover a more geometric description of $\Xi$ in the future.

\section{A two-sided Coxeter complex}

Fix a Coxeter system $(W, S)$ with $|S|=n$. We call the elements $s \in S$ the simple generators of $W$. Every element $w \in W$ can be written as a product of elements in $S, w=s_{1} \cdots s_{k}$, and if this expression is minimal, we say the length of $w$ is $k$, denoted $\ell(w)=k$. An expression of minimal length is called a reduced expression.

The weak order is an important partial order on $W$. The weak order comes in two equivalent types: "left" and "right" weak order. The left weak order is the transitive closure of the relations $u<_{L} s u$, where $s \in S$ and $\ell(s u)=\ell(u)+1$. Similarly, the covers for the right weak order are of the form $u<_{R} u s$ where $\ell(u s)=\ell(u)+1$. We denote the left weak order by $u \leq_{L} v$ and the right weak order by $u \leq_{R} v$. The "two-sided" weak order is the transitive closure of both types of cover relations, denoted by $u \leq_{L R} v$.

The left (resp. right) descent set of an element $w$ is the set of all simple generators that correspond to downward covers in left (resp. right) weak order. We denote the left and right descent sets by $\operatorname{Des}_{L}(w)$ and $\operatorname{Des}_{R}(w)$, respectively, i.e.,

$$
\operatorname{Des}_{L}(w)=\left\{s \in S: s w<_{L} w\right\} \text { and } \operatorname{Des}_{R}(w)=\left\{s \in S: w s<_{R} w\right\} .
$$

We define the corresponding ascent sets as the complements of the descent sets in $S$ :

$$
\operatorname{Asc}_{L}(w)=S-\operatorname{Des}_{L}(w)=\left\{s \in S: s w>_{L} w\right\},
$$

and

$$
\operatorname{Asc}_{R}(w)=S-\operatorname{Des}_{R}(w)=\left\{s \in S: w s>_{R} w\right\} .
$$

Given a subset of simple generators, $J \subseteq S$, let $W_{J}$ denote the group generated by the elements of $J$, i.e., $W_{J}=\langle s: s \in J\rangle$. This group is a Coxeter group in its own right, and we call such a subgroup 
a standard parabolic subgroup. The Coxeter complex arises when considering the quotients of the form $W / W_{J}$. As such, the faces of the Coxeter complex are identified with left cosets of parabolic subgroups $w W_{J}$. For our two-sided analogue we consider elements from all double quotients $W_{I} \backslash W / W_{J}$, so the faces will be related to double cosets of parabolic subgroups $W_{I} w W_{J}$, where $I$ and $J$ are subsets of $S$.

An essential fact about cosets of parabolic subgroups is that each coset $w W_{J}$ has a unique element of minimal length, call it $u$, such that $J \subseteq \operatorname{Asc}_{R}(u)$, or $\operatorname{Des}_{R}(u) \subseteq S-J$. In fact, the same is true for double cosets, and we record this in the following lemma, which can be found in [7, Chapter 4, Exercise $1.3]$.

Lemma 1 Each double coset $W_{I} w W_{J}$ has a unique element of minimal length, call it $u$, such that

$$
I \subseteq \operatorname{Asc}_{L}(u) \quad \text { and } \quad J \subseteq \operatorname{Asc}_{R}(u) .
$$

Moreover, for each $v \in W_{I} w W_{J}$, $u$ is below $v$ in the two-sided weak order: $u \leq_{L R} v$.

Let ${ }^{I} W^{J}$ denote the set of minimal representatives for $W_{I} \backslash W / W_{J}$, i.e.,

$$
{ }^{I} W^{J}=\left\{w \in W: I \subseteq \operatorname{Asc}_{L}(w) \text { and } J \subseteq \operatorname{Asc}_{R}(w)\right\} .
$$

If $I=\emptyset$ we have ${ }^{\emptyset} W^{J}=W^{J}$ is the set of left coset representatives.

With the lemma in mind, we could just as easily replace the cosets $w W_{J}$ in the definition of $\Sigma$ with pairs $(w, J)$ such that $w \in W^{J}$. Extending this idea, we make the following definition.

Let

$$
\Xi=\left\{(I, w, J): I, J \subseteq S \text { and } w \in{ }^{I} W^{J}\right\} .
$$

We partially order the elements of $\Xi$ by reverse inclusion of the index sets $I$ and $J$ as well as the corresponding double coset, i.e.,

$$
(I, w, J) \leq \Xi\left(I^{\prime}, w^{\prime}, J^{\prime}\right) \quad \text { if and only if } \quad\left\{\begin{array}{l}
I \supseteq I^{\prime} \\
J \supseteq J^{\prime}, \text { and } \\
W_{I} w W_{J} \supseteq W_{I^{\prime}} w W_{J^{\prime}}
\end{array}\right.
$$

We will refer to the $\Xi$ as the two-sided Coxeter complex.

\section{$2.1 \Xi$ is a balanced boolean complex}

The maximal elements in $\Xi$ are those of the form $(\emptyset, w, \emptyset)$, and there is a unique minimum, $(S, e, S)$. The rank one elements are those of the form $(S-\{i\}, e, S)$ and $(S, e, S-\{k\})$, i.e., those obtained by omitting a single element from $S$ on either the left or on the right.

We will now prove that lower intervals in the poset $\Xi$ are isomorphic to boolean algebras. Since the face poset of a simplex is the boolean algebra on its vertex set, a poset with this property is known as a simplicial poset, or as a boolean complex.

Proposition 1 The poset $\Xi$ is a simplicial poset. In particular, the interval below the element $(I, w, J) \in$ $\Xi$ isomorphic to the set of all subsets of $(S-I) \times(S-J)$. 
Proof: Fix an element $F=(I, w, J)$ of $\Xi$ and consider any element below $F$ in the partial order, i.e., suppose we have an element $\left(I^{\prime}, w^{\prime}, J^{\prime}\right) \leq_{\Xi} F$. Then by definition, $S \supseteq I^{\prime} \supseteq I$ and $S \supseteq J^{\prime} \supseteq J$, so $\left(I^{\prime}-I, J^{\prime}-J\right)$ is an element of $(S-I) \times(S-J)$.

To finish the proof we must show that every pair of subsets $\left(I^{\prime}-I, J^{\prime}-J\right)$ in $(S-I) \times(S-J)$ corresponds to a unique element below $F$.

Suppose $\left(I^{\prime}-I, J^{\prime}-J\right)$ is a pair of subsets in $(S-I) \times(S-J)$, i.e., $S \supseteq I^{\prime} \supseteq I$ and $S \supseteq$ $J^{\prime} \supseteq J$. If $C=W_{I^{\prime}} v W_{J^{\prime}}$ is a coset that contains $W_{I} w W_{J}$, then in particular $w \in C$ and we can write $C=W_{I^{\prime}} w W_{J^{\prime}}$. Thus for fixed $I^{\prime}$ and $J^{\prime}$, there is one such coset. By Lemma 1 there exists a unique element $w^{\prime} \in C$ such that $\operatorname{Des}_{L}\left(w^{\prime}\right) \subseteq S-I^{\prime}$ and $\operatorname{Des}_{R}\left(w^{\prime}\right) \subseteq S-J^{\prime}$. This identifies the unique triple $G=\left(I^{\prime}, w^{\prime}, J^{\prime}\right)$ such that $G \leq_{\Xi} F$, completing the proof.

Proposition 1 means that each element of $\Xi$ can be thought of as an abstract simplex. As such, we will refer to the elements as faces. We say a face $(I, w, J)$ is represented by $w$. While each face of $\Xi$ is a simplex, it is not a simplicial complex, since distinct faces may share the same vertex set. In fact, we will see that for any $(W, S), \Xi$ has the property that every facet (maximal face) has the same vertex set.

The dimension of a face is given by one less than its rank in the poset, i.e., if $F=(I, w, J)$,

$$
\operatorname{dim} F=|S-I|+|S-J|-1
$$

In particular, if $|S|=n$, then $\Xi$ has $2 n$ vertices, each of the form $(S-\{i\}, e, S)$ or $(S, e, S-\{j\})$. The facets are of the form $(\emptyset, w, \emptyset)$ and so $\operatorname{dim} \Xi=2 n-1$. Since there are only $2 n$ vertices, we see that $\Xi$ is trivially balanced, i.e., we can assign colors $2 n$ colors to the vertices so that no face has two vertices of the same color. Along with Proposition 1 we have now established part (1) of Theorem 2

\section{$2.2 \Sigma$ is a relative subcomplex of $\Xi$}

We have already mentioned that maximal faces of $\Xi$ are in bijection with elements of $W$. Let us denote the facet corresponding to an element $w$ by $F_{w}=(\emptyset, w, \emptyset)$. If we consider only adding elements to the left index set (or only adding elements to the right) we get a subposet of $\Xi$ that corresponds to a facet of the usual Coxeter complex, i.e., as posets

$$
\left[(S, e, \emptyset), F_{w}\right] \cong\left[(\emptyset, e, S), F_{w}\right] \leftrightarrow[(e, S),(w, \emptyset)] \in \Sigma
$$

Taking the union of all such intervals (choosing the left or right version) we get a full copy of $\Sigma$ as an upper order ideal inside of $\Xi$.

$$
\begin{aligned}
\Sigma=\left\{(w, J): J \subseteq S, w \in W^{J}\right\} & \cong\left\{(\emptyset, w, J): J \subseteq S, w \in{ }^{\emptyset} W^{J}\right\} \\
& =\{F \in \Xi:(\emptyset, e, S) \leq \Xi F\}
\end{aligned}
$$

To phrase this result another way, we say that $\Sigma$ is a relative subcomplex of $\Xi$. This establishes part (2) of Theorem 2

\section{3 $\Xi$ is partitionable}

We can notice that the faces represented by a given element $w$ form an interval in $\Xi$ whose maximal element is a maximum of $\Xi$. That is, let $R_{w}=\left(\operatorname{Asc}_{L}(w), w, \operatorname{Asc}_{R}(w)\right)$, which we call the restriction of 
$w$. Then the interval $\left[R_{w}, F_{w}\right]$ in $\Xi$ consists of all faces represented by $w$, and moreover this interval is boolean:

$$
\begin{aligned}
{\left[R_{w}, F_{w}\right] } & =\left\{(I, w, J): I \subseteq \operatorname{Asc}_{L}(w), J \subseteq \operatorname{Asc}_{R}(w)\right\} \\
& \cong \operatorname{Asc}_{L}(w) \times \operatorname{Asc}_{R}(w)
\end{aligned}
$$

The union of all such intervals partitions the faces of $\Xi$, i.e.,

$$
\Xi=\bigcup_{w \in W}\left[R_{w}, F_{w}\right]
$$

and this union is disjoint. Moreover, since each interval in the partition is an upper ideal isomorphic to a boolean algebra, $\Xi$ is partitionable in the topological sense as well. See [20] for the relevant definition.

\section{$2.4 \Xi$ is shellable}

Before describing how to find a shelling of $\Xi$, we first make the following simple observation. If $(I, u, J)$ is a face of $\Xi$ below the face $\left(I^{\prime}, v, J^{\prime}\right)$, then in particular $W_{I^{\prime}} v W_{J^{\prime}} \subseteq W_{I} u W_{J}$, and $v \in W_{I} u W_{J}$. But by Lemma 1 this means $u$ is below $v$ in the two-sided weak order.

Observation 1 If $(I, u, J) \leq_{\Xi}\left(I^{\prime}, v, J^{\prime}\right)$, then $u \leq_{L R} v$.

From this simple observation it follows that any choice of linear extension of the two-sided weak order for $W$ is a shelling order for $\Xi$. First recall the definition of a shelling of a boolean complex. This is an ordering of the facets $F_{1}, F_{2}, \ldots$ such that the intersection of the boundary of each new facet with the union of the boundaries of the prior facets is a pure codimension one complex. That is, for each $k$, we must show

$$
\partial F_{k} \cap\left(\bigcup_{i=1}^{k-1} \partial F_{i}\right)
$$

is a pure codimension one complex. Here $\partial F_{k}$ denotes the boundary of $F_{k}$, i.e., all proper faces of $F_{k}$.

Consider all the codimension one faces of the facet $F_{w}=(\emptyset, w, \emptyset)$. These come in four types:

$\bullet(\{s\}, s w, \emptyset)$ if $s \in \operatorname{Des}_{L}(w) \quad \bullet(\emptyset, w s,\{s\})$ if $s \in \operatorname{Des}_{R}(w)$

$\bullet(\{s\}, w, \emptyset)$ if $s \in \operatorname{Asc}_{L}(w) \quad \bullet(\emptyset, w,\{s\})$ if $s \in \operatorname{Asc}_{R}(w)$

In the first two cases, the elements $s w$ and $w s$ are below $w$ in the two-sided weak order. If we order the facets of $\Xi$ according to a linear extension of the two-sided weak order:

$$
F_{w_{1}}, F_{w_{2}}, \ldots, F_{w_{k}}, F_{w}, \ldots,
$$

then the intersection of $F_{w}$ with the union of the prior facets is given by those faces below $F_{w}$ in $\Xi$ that are not represented by $w$, i.e.,

$$
\partial F_{w} \cap\left(\bigcup_{i=1}^{k} \partial F_{w_{i}}\right)=\bigcup_{\substack{s \in \operatorname{Des}_{L}(w) \\ t \in \operatorname{Des}_{R}(w)}}[(S, e, S),(\{s\}, s w, \emptyset)] \cup[(S, e, S),(\emptyset, w t,\{t\})] .
$$

Because all maximal faces in the union have codimension one, we have proved the following proposition.

Proposition 2 (Shelling order) Any linear extension of the two-sided weak order on $W$ is a shelling order for $\Xi$.

This proves part (3) of Theorem 2 


\subsection{Consequences of shelling}

Let us say a bit more about the codimension one faces of $\Xi$. These are of the form $(\{s\}, w, \emptyset)$ or $(\emptyset, w,\{t\})$. Without loss of generality, consider the first case. The double coset here has only two elements: $W_{\{s\}} w W_{\emptyset}=\{w, s w\}$, so the face $(\{s\}, w, \emptyset)$ is only contained in the facets $(\emptyset, w, \emptyset)$ and $(\emptyset, w s, \emptyset)$. This shows that $\Xi$ is what is called a thin complex (also sometimes known as a pseudomanifold).

Results of Björner ([5, Theorem 1.5] and [4, Proposition 4.3]) tells us about thin, shellable complexes. If the complex is infinite, it is contractible. If the complex is finite it is a sphere. Thus (by passing to the barycentric subdivision so we get an honest simplicial compex) we obtain the following corollary, establishing parts (4) and (5a) of Theorem 2

Corollary 1 Each codimension one face of $\Xi$ is contained in exactly two facets, and hence:

- $\Xi$ is contractible when $W$ is infinite,

- $\Xi$ is a sphere when $W$ is finite.

Before we move on to prove the remaining part of Theorem 2, we include some remarks.

Remark 1 A first guess to define a two-sided Coxeter complex is to consider the set of all double cosets $W_{I} w W_{J}$, ordered by reverse inclusion. Such a poset does indeed exist, but it is difficult to analyze. It is not even obvious when this poset is ranked. For one thing, there are many subtle equalities of cosets, e.g., with $w$ fixed, we might have $W_{I} w W_{J}=W_{I^{\prime}} w W_{J^{\prime}}$ and yet $I \neq I^{\prime}$ or $J \neq J^{\prime}$. For an extreme case, notice that for any $I \subseteq J$, we have $W_{I} e W_{J}=W_{J}$. Enumeration of the number of distinct double cosets is the topic of ongoing work of Billey, Konvalinka, Petersen, Slofstra, and Tenner [3].

Remark 2 If we fix a choice of $J$ and $K$, we can restrict the Bruhat order on $W$ to give a partial ordering on the elements ${ }^{J} W^{K}$, or on the double quotient $W_{J} \backslash W / W_{K}$. Stembridge gives a geometric construction of this partial order in terms of root systems [21]. Diaconis and Gangolli did the same in the case of the symmetric group, realized as a partial order on contingency tables with prescribed row and column sums [10].

\section{Face enumeration for finite $W$}

Throughout this section we assume $W$ is finite and fix an ordering on the generating set, $S=\left\{s_{1}, \ldots, s_{n}\right\}$. In this way we can identify subsets of $S$ with subsets of $[n]:=\{1,2, \ldots, n\}$. Let $x_{1}, \ldots, x_{n}$ and $y_{1}, \ldots, y_{n}$ be indeterminates. If $I \subseteq[n]$, let $x_{I}=\prod_{i \in I} x_{i}$, and similarly for $y_{I}$.

For a face $F=(I, w, J)$ in $\Xi$, the face monomial for $F$ is

$$
m(F)=x_{[n]-I} y_{[n]-J}=\prod_{i \in[n]-I} x_{i} \prod_{j \in[n]-J} y_{j} .
$$

Notice this encodes the color of the face $F$; the $x$ variables encode the left sided vertices, the $y$ variables encode the right sided vertices.

Let $f(\mathbf{x}, \mathbf{y})=f\left(x_{1}, \ldots, x_{n}, y_{1}, \ldots, y_{n}\right)$ denote the generating function for colors of faces, i.e.,

$$
f(\mathbf{x}, \mathbf{y})=\sum_{F \in \Xi} m(F)=\sum_{I, J} f_{I, J} x_{I} y_{J} .
$$


Notice that the coefficient $f_{I, J}$ is the number of faces $(S-I, w, S-J)$, i.e., it counts the cardinality of the corresponding double quotient:

$$
f_{I, J}=\left|{ }^{S-I} W^{S-J}\right|=\left|W_{S-I} \backslash W / W_{S-J}\right|=\left|\left\{w \in W: \operatorname{Des}_{L}(w) \subseteq I, \operatorname{Des}_{R}(w) \subseteq J\right\}\right| .
$$

Now define the quantities

$$
h_{I, J}=\sum_{\substack{K \subseteq I \\ L \subseteq J}}(-1)^{|I-K|+|J-L|} f_{K, L}=\left|\left\{w \in W: \operatorname{Des}_{L}(w)=I, \operatorname{Des}_{R}(w)=J\right\}\right|,
$$

and the corresponding generating function $h(\mathbf{x}, \mathbf{y})=h\left(x_{1}, \ldots, x_{n}, y_{1}, \ldots, y_{n}\right)$ by

$$
h(\mathbf{x}, \mathbf{y})=\sum_{I, J} h_{I, J} x_{I} y_{J},=\sum_{w \in W} x_{\operatorname{Des}_{L}(w)} y_{\operatorname{Des}_{R}(w)} .
$$

Using the partitioning of faces of $\Xi$ given in (1), it is straightforward to check that

$$
\begin{aligned}
f(\mathbf{x}, \mathbf{y}) & =\sum_{w \in W} \sum_{R_{w} \leq F \leq F_{w}} m(F), \\
& =\prod_{i=1}^{n}\left(1+x_{i}\right)\left(1+y_{i}\right) \cdot h\left(\frac{x_{1}}{1+x_{1}}, \ldots, \frac{x_{n}}{1+x_{n}}, \frac{y_{1}}{1+y_{1}}, \ldots, \frac{y_{n}}{1+y_{n}}\right) .
\end{aligned}
$$

That is, we obtain the $f$-polynomial as a multiple of a certian specialization of the $h$-polynomial. Putting identity (2) the other way around, we can write

$$
h(\mathbf{x}, \mathbf{y})=\prod_{i=1}^{n}\left(1-x_{i}\right)\left(1-y_{i}\right) \cdot f\left(\frac{x_{1}}{1-x_{1}}, \ldots, \frac{x_{n}}{1-x_{n}}, \frac{y_{1}}{1-y_{1}}, \ldots, \frac{y_{n}}{1-y_{n}}\right) .
$$

Setting $x_{i}=x$ and $y_{j}=y$, the $h$-polynomial specializes to

$$
h(x, y)=\sum_{w \in W} x^{\operatorname{des}_{L}(w)} y^{\operatorname{des}_{R}(w)}
$$

In other words, the polynomial $h(x, y)$ is a "two-sided" Eulerian polynomial. This establishes the claim in part (5b) of Theorem 2.

We also comment that setting $x_{i}=y_{i}=x$ in $f$ or $h$ would recover the usual $f$ - and $h$-polynomials of $\Xi$, while setting $x_{i}=x$ and $y_{i}=1$ would recover the usual $f$ - and $h$-polynomials of $\Sigma$.

Having established Theorem 2 in full, we now turn to some related questions and observations.

\section{Two-sided Eulerian polynomials}

With finite $W$, we can define two-sided $W$-Eulerian polynomial, denoted $W(x, y)$, as the joint distribution of left and right descents:

$$
W(x, y)=\sum_{w \in W} x^{\operatorname{des}_{L}(w)} y^{\operatorname{des}_{R}(w)}=\sum_{0 \leq i, j \leq n}\left\langle\begin{array}{l}
W \\
i, j
\end{array}\right\rangle x^{i} y^{j},
$$


where $\left\langle{ }_{i, j}^{W}\right\rangle$ denotes the number of elements in $W$ with $i$ left descents and $j$ right descents. We call $\left\langle{ }_{i, j}^{W}\right\rangle$ a two-sided $W$-Eulerian number.

For example if $W=E_{6}$, we have the following array of two-sided Eulerian numbers:

$$
\left.\left[\left\langle E_{6}\right\rangle\right]_{i, j}\right]_{0 \leq i, j \leq 6}=\left[\begin{array}{rrrrrrl}
1 & 0 & 0 & 0 & 0 & 0 & 0 \\
0 & 232 & 584 & 389 & 64 & 3 & 0 \\
0 & 584 & 4785 & 5440 & 1310 & 64 & 0 \\
0 & 389 & 5440 & 13270 & 5440 & 389 & 0 \\
0 & 64 & 1310 & 5440 & 4785 & 584 & 0 \\
0 & 3 & 64 & 389 & 584 & 232 & 0 \\
0 & 0 & 0 & 0 & 0 & 0 & 1
\end{array}\right] .
$$

For the family of type $A_{n}$, these numbers were first studied by Carlitz, Roselle, and Scoville [9], but have been recently revisited by the author [17] and Visontai [23] (who also discussed type $B_{n}$ Coxeter groups). The recent interest in these polynomials stems from a conjecture of Gessel that we will now describe and generalize to all Coxeter groups.

To state Gessel's conjecture, one must first make note of certain symmetries in the two-sided Eulerian numbers. Notice that the map $w \mapsto w^{-1}$ swaps left and right descents, $\operatorname{des}_{L}(w)=\operatorname{des}_{R}\left(w^{-1}\right)$, so we get symmetry in $i$ and $j$ :

$$
\left\langle\begin{array}{l}
W \\
i, j
\end{array}\right\rangle=\left\langle\begin{array}{l}
W \\
j, i
\end{array}\right\rangle
$$

Also recall that left multiplication by the long element $w_{0}$ is an anti-automorphism of the weak order: i.e., $w s<_{L} w$ if and only if $w_{0} w s>_{L} w_{0} w$, and similarly for the right weak order. Thus if $|S|=n$, $\operatorname{des}_{L}\left(w_{0} w\right)=n-\operatorname{des}_{L}(w)$ and $\operatorname{des}_{R}\left(w_{0} w\right)=n-\operatorname{des}_{R}(w)$. Hence we have

$$
\left\langle\begin{array}{l}
W \\
i, j
\end{array}\right\rangle=\left\langle\begin{array}{c}
W \\
n-i, n-j
\end{array}\right\rangle
$$

Phrasing symmetries (4) and (5), we have the following observation about the two-sided $W$-Eulerian polynomials.

Observation 2 For any finite Coxeter group $W$ of rank $n$,

1. $W(x, y)=W(y, x)$, and

2. $W(x, y)=x^{n} y^{n} W(1 / x, 1 / y)$.

Integer polynomials that possess symmetries as in Observation 2 have an expansion in the following basis:

$$
\Gamma_{n}=\left\{(x y)^{a}(x+y)^{b}(1+x y)^{n-2 a-b}\right\}_{0 \leq 2 a+b \leq n} .
$$

The generalized Gessel conjecture is that the two-sided Eulerian polynomials expand positively in this basis. This generalizes the univariate "gamma basis" whose study has led to many interesting results. See [8, 13, 19] and [18, Chapter 4].

Conjecture 1 (Generalized Gessel's conjecture) For any finite Coxeter group $W$, there exist nonnegative integers $\gamma_{a, b}^{W}$ such that

$$
W(x, y)=\sum_{0 \leq 2 a+b \leq n} \gamma_{a, b}^{W}(x y)^{a}(x+y)^{b}(1+x y)^{n-2 a-b} .
$$




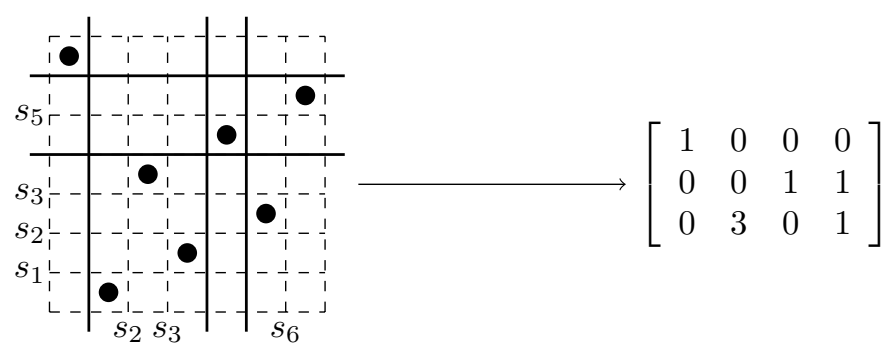

Fig. 1: A double coset in $A_{6}$ mapping to a contingency table in $\Xi(7)$.

The univariate version of this claim (i.e., with $x=1$ ) is true, though there is no case-free proof. See [12, 16, 22] and also [18, Theorem 11.2].

Remark 3 Very recently, the author was informed that Gessel's original conjecture (for $W=A_{n}=$ $S_{n+1}$ ) was proved by Lin [15]. The method of proof seems to have been a careful induction argument using a recurrence for the $\gamma_{a, b}^{A_{n}}$ given by Visontai [23]. The other cases have been verified for small rank $(n \leq 8)$. Type $B_{n}$ is governed by similar combinatorics, so perhaps a similar induction proof can be found. In all cases, it would be nice to know what the $\gamma_{a, b}^{W}$ count.

\section{Contingency tables}

Throughout this section we consider the special case where $W=S_{n}$ is the symmetric group. As shown in Diaconis and Gangolli [10], for fixed $I$ and $J$ the double cosets $W_{I} w W_{J}$ are in bijection with arrays of nonnegative integers with prescribed row and column sums. Our contribution here is to say that the partial order on $\Xi$ translates to refinement order on these arrays.

To see how this connection is made, we draw double cosets as diagrams of "balls in boxes." First, we draw permutations as two-dimensional arrays. If $w(i)=j$ we draw a ball in column $i$ (left to right), row $j$ (bottom to top). We then insert some vertical and horizontal bars in gaps between balls. The symmetric group acts on the left by permuting rows that are not separated by a bar. It acts on the right by permuting columns not separated by a bar.

The left and right descents are easily seen in this picture: left descents correspond to adjacent rows in which the ball in the top row is to the left of the ball in the bottom row. Right descents correspond to adjacent columns in which the ball in the first column is higher than the ball in the second. For example, $w=7142536$ is drawn in Figure 1 . We can see $\operatorname{des}_{L}(w)=\{3,6\}$ and $\operatorname{des}_{R}(w)=\{1,3,5\}$.

To indicate a double parabolic coset $W_{I} w W_{J}$, we draw solid horizontal bars in gaps that correspond to $S-I$ and solid vertical bars in gaps that correspond to $S-J$. In Figure $1 . I=\left\{s_{1}, s_{2}, s_{3}, s_{5}\right\}$ and $J=\left\{s_{2}, s_{3}, s_{6}\right\}$. The minimal representative for the double coset corresponds to the permutation obtained by sorting the balls in increasing order from left to right and from bottom to top. The minimal representative for the coset illustrated in Figure 1 would then be $u=7123546$. Notice that both the left descents and right descents of $u$ occur in barred positions.

We can map a balls-in-boxes diagram to an array of nonnegative integers by merely counting the number of balls in each box. See Figure 1 . Let $\Xi(n)$ denote the set of all such arrays, which are known as two-way contingency tables. More precisely, define $\Xi(n)$ to be the set of all nonnegative integer arrays whose entries sum to $n$ and whose row sums and column sums are positive. 
To move up in the partial order on $\Xi$, we refine our balls and boxes picture by inserting more bars. On the contingency table side, this means our arrays get more rows and columns. Each cover relation corresponds to adding or deleting a single bar, so rank is given by the total number of bars. A balls-inboxes picture with $k$ horizontal bars and $l$ vertical bars will correspond to a $(k+1) \times(l+1)$ contingency table. Note that maximal contingency tables are permutation matrices.

Downward covers in the partial order correspond to removing a single bar from the balls in boxes picture, which therefore adds all the entries in two adjacent rows or two adjacent columns of the corresponding contingency tables.

Proposition 3 The two-sided Coxeter complex of the symmetric group $S_{n}$ is isomorphic to $\Xi(n)$ under refinement order.

Remark 4 The dual of the type $A_{n}$ Coxeter complex is the permutahedron, which plays an interesting role in the study of combinatorial Hopf algebras, such as the Malvenuto-Reutenauer algebra and the algebra of quasisymmetric functions. See work of Aguiar and Sottile, for example [2].

Suggestively, two-way contingency tables provide an indexing set for a Hopf algebra known as the set of matrix quasisymmetric functions, which gives several well-known combinatorial Hopf algebras (e.g., quasisymmetric functions, noncommutative symmetric functions) as subalgebras or quotients. See work of Duchamp, Hivert, and Thibon [11. Section 5]. It would interesting to explore whether $\Xi(n)$ might play a role for the matrix quasisymmetric functions similar to the role the permutahedron plays for the Malvenuto-Reutenauer algebra.

Remark 5 One can generalize from $\Xi(n)$ to $\Xi(k ; n)$, the set of all $k$-way contingency tables. These are $k$-dimensional arrays of nonnegative integers whose entries sum to $n$ such that all their marginal sums are positive. Refinement ordering makes $\Xi(k ; n)$ a thin, shellable simplicial poset. (There is a natural generalization of weak order whose linear extensions provide shelling orders.) Thus $\Xi(k ; n)$ is also a sphere. Its dimension is $k(n-1)-1$ and it has $(n !)^{k-1}$ facets.

\section{Acknowledgements}

The author would like to thank John Stembridge for early conversations about possible topological interpretations for the two-sided Eulerian polynomials.

\section{References}

[1] P. Abramenko and K. S. Brown, "Buildings. Theory and applications.” Graduate Texts in Mathematics, 248. Springer, New York, 2008.

[2] M. Aguiar and F. Sottile, Structure of the Malvenuto-Reutenauer Hopf algebra of permutations, Adv. Math. 191 (2005), 225-275.

[3] S. Billey, M. Konvalinka, T. K. Petersen, W. Slofstra, and B. E. Tenner, Parabolic double cosets in Coxeter groups, in preparation (2015).

[4] A. Björner, Posets, regular CW complexes and Bruhat order, European J. Combin. 5 (1984), 7-16.

[5] A. Björner, Some combinatorial and algebraic properties of Coxeter complexes and Tits buildings, Adv. in Math. 52 (1984), 173-212. 
[6] A. Björner and F. Brenti, "Combinatorics of Coxeter groups." Graduate Texts in Mathematics, 231. Springer, New York, 2005.

[7] N. Bourbaki, "Lie groups and Lie algebras. Chapters 4-6." Translated from the 1968 French original by Andrew Pressley. Elements of Mathematics (Berlin). Springer-Verlag, Berlin, 2002.

[8] P. Brändén, Actions on permutations and unimodality of descent polynomials, European J. Combin. 29 (2008), 514-531.

[9] L. Carlitz, D. P. Roselle, and R. A. Scoville, Permutations and sequences with repetitions by number of increases, J. Combinatorial Theory 1 (1966), 350-374.

[10] P. Diaconis and A. Gangolli, Rectangular arrays with fixed margins. Discrete probability and algorithms (Minneapolis, MN, 1993), 15-41, IMA Vol. Math. Appl., 72, Springer, New York, 1995.

[11] G. Duchamp, F. Hivert, and J.-Y. Thibon, Noncommutative symmetric functions. VI. Free quasisymmetric functions and related algebras. Internat. J. Algebra Comput. 12 (2002), no. 5, 671-717.

[12] D. Foata and M.-P. Schützenberger, "Théorie géométrique des polynômes eulériens." (French) Lecture Notes in Mathematics, Vol. 138 Springer-Verlag, Berlin-New York 1970.

[13] S. Gal, Real root conjecture fails for five- and higher-dimensional spheres, Discrete Comput. Geom. 34 (2005), 269-284.

[14] J. Humphreys, "Reflection groups and Coxeter groups," Cambridge Studies in Advanced Mathematics, 29. Cambridge University Press, Cambridge, 1990.

[15] Z. Lin, personal communication (2015).

[16] T. K. Petersen, Enriched P-partitions and peak algebras, Adv. Math. 209 (2007), 561-610.

[17] T. K. Petersen, Two-sided Eulerian numbers via balls in boxes, Math. Mag. 86 (2013), no. 3, 159176.

[18] T. K. Petersen, "Eulerian numbers," Birkhäuser Advanced Texts Basler Lehrbücher. Springer, New York, 2015.

[19] A. Postnikov, V. Reiner, L. Williams, Faces of generalized permutohedra, Doc. Math. 13 (2008), 207-273.

[20] R. P. Stanley, "Combinatorics and commutative algebra," Second edition. Progress in Mathematics, 41. Birkhäuser Boston, Inc., Boston, MA, 1996.

[21] J. R. Stembridge, Tight quotients and double quotients in the Bruhat order, Electron. J. Combin. 11 (2004/06), Research Paper 14, 41 pp.

[22] J. R. Stembridge, Coxeter cones and their h-vectors, Adv. Math. 217 (2008), 1935-1961.

[23] M. Visontai, Some remarks on the joint distribution of descents and inverse descents, Electron. J. Combin. 20 (2013), Paper 52, 12 pp. 